\title{
On the Treatment of Incomparability in Ordering Semantics and Premise Semantics
}

\author{
Eric Swanson \\ ericsw@umich.edu \\ This is the penultimate version. \\ Please consult the official version in the Journal of Philosophical Logic.
}

\begin{abstract}
In his original semantics for counterfactuals, David Lewis presupposed that the ordering of worlds relevant to the evaluation of a counterfactual admitted no incomparability between worlds. He later came to abandon this assumption. But the approach to incomparability he endorsed makes counterintuitive predictions about a class of examples circumscribed in this paper. The same underlying problem is present in the theories of modals and conditionals developed by Bas van Fraassen, Frank Veltman, and Angelika Kratzer. I show how to reformulate all these theories in terms of lower bounds on partial preorders, conceived of as maximal antichains, and I show that treating lower bounds as cutsets does strictly better at capturing our intuitions about the semantics of modals, counterfactuals, and deontic conditionals.
\end{abstract}

Keywords Modals · Counterfactuals · Incomparability · Ordering semantics ·

Premise semantics

In his original semantics for counterfactuals, David Lewis presupposed

\section{COMPARABILITY:}

For any world of evaluation $i$, the ordering of worlds $\lesssim_{i}$ relevant to the evaluation of a counterfactual ' $\mathcal{A}>\mathcal{C}$ ' makes any two worlds that are $\lesssim_{i}$-comparable to $i$ also $\lesssim_{i}$-comparable to each other. ${ }^{1}$

COMPARABILITy should not be taken lightly. In assuming that COMPARABILITY is true one makes strong commitments about the ways in which worlds are and can be compared and ordered, about the information carried by conversational context, and about the proper treatment of prima facie inconsistencies in modal bases and ordering sources. Unfortunately the semantic theory that Lewis developed in an effort to abandon COMPARABILITY makes counterintuitive predictions about a class of

Thanks to Thony Gillies, Jim Pryor, Jason Stanley, Brian Weatherson, and the audience at the 2009 Rutgers Semantics Workshop for helpful discussion. Thanks especially to Sarah Moss, Robert van Rooij, Rich Thomason, and an anonymous reviewer for discussion and comments on earlier drafts.

${ }^{1}$ Sections 2.3 and 6.1 of Lewis 1973 are especially forthright on this point. 
examples circumscribed in this paper. The standard versions of ordering semantics and premise semantics for modals also make counterintuitive predictions about that class of examples. This paper develops alternative semantic theories for modals and counterfactuals and shows that they strictly improve on the standard theories.

\section{Background}

It is helpful to start with modals; I focus on Angelika Kratzer's ordering semantics because of its influence and familiarity. A bit of notation and terminology:

Definition 1. A relation is a preorder iff it is conditionally reflexive ${ }^{2}$ and transitive.

Definition 2. A preorder $\lesssim$ totally preorders a set $S$ iff $\forall x \forall y((x \in S \wedge y \in S) \rightarrow(x \lesssim$ $y \vee y \lesssim x)$ ).

A non-total preorder may also be called 'merely partial.' Some uses of "partial order" in the literature (e.g., some uses in LewIS 1981) should be read as 'partial preorder', following the convention of LEWIS 1973, 48.

Definition 3. $\lesssim_{i}$ (read 'is at least as good as at the world of evaluation $i$ ') is a partial preorder of a set of worlds.

Definition 4. $S_{\varsigma_{i}}$ (read 'the set of worlds comparable to $i$ ') is $\left\{w: w \lesssim_{i} i \vee i \lesssim_{i} w\right\}$.

Definition $5 .<_{i}$ (read 'is better than at the world of evaluation $i$ ') is a strict partial order such that $\forall x \forall y\left(x<_{i} y \leftrightarrow\left(x \lesssim_{i} y \wedge y \mathbb{k}_{i} x\right)\right)$.

I am compelled by Kratzer's hypothesis that although different uses of a given modal can be used to target different flavors of modality, "there is something in the meaning [of that modal] ... which stays invariable" $(1977,340)$. It is because I endorse this hypothesis that I read $\lesssim_{i}$ and $<_{i}$ as 'is at least as good as' and 'is better than': I think it is helpful to characterize the preorders relevant to different modal flavors as generally as possible. The qualitative terms 'good' and 'better' are apt descriptions of all sorts of preorders, including those relevant to counterfactuals, epistemic modals, and (most naturally) deontic and bouletic modals. Those who prefer more familiar talk of 'closeness' and 'resemblance' for counterfactuals may read 'is better than' as 'more closely resembles the world of evaluation. ${ }^{3}$ But it is easy to be misled by 'close,'

\footnotetext{
${ }^{2} \lesssim$ is conditionally reflexive iff $\forall x(\exists y(x \lesssim y \vee y \lesssim x) \rightarrow x \lesssim x)$. Preorders are usually defined as reflexive and transitive relations; I am loosening the definition slightly to allow for the possibility that the relevant preorder takes no stand whatsoever on some accessible worlds.

${ }^{3}$ For epistemic modals one may read 'is better than' as 'reflects more information,' where the amount of information is measured by strict set inclusion.
} 
'closer,' 'nearby', and other spatial metaphors into imputing too much structure to a preorder, assuming perhaps that it has properties of a metric space-for example, that it is total, or that there is an ideal that is a finite 'distance' away from any point. 'At least as good as' and 'is better than' are quite helpful in this respect: it is not counterintuitive to think that two situations might each be better than a third and yet incomparable to each other, or to think that one situation might be better than another but not better 'by' any quantifiable factor or amount. Moreover, it may be that in philosophers' discussions of counterfactuals metaphors of distance have become counterproductively intertwined with questions about the logic and semantics of counterfactuals. Even if Lewis is wrong about which preorders are relevant to the evaluation of counterfactuals (see, e.g., HÁJEK 2009), ordering semantics might still be the right semantics for counterfactuals. Abandoning distance metaphors helps underscore this point.

' $\lesssim$ ' and ' $<$ ' are subscripted with ' $i$ ' to index them to the world of evaluation. According to standard versions of ordering semantics for modals, to say how things might be, for all we know, is to make claims about how things are at possible worlds consistent with what we know. As a result, which preorders matter to the evaluation of a given sentence sometimes depends on how things would have been if things had been different. For example, on standard ordering semantics for 'might' and 'is permissible,' the claim that

(1) It might be that lying is permissible.

entails that some world $w$ consistent with what is known makes lying permissible according to the deontic preorder at $w$. But a speaker who asserts (1) may take no stand on whether $w$ is the actual world. For (1) to be true there must be some epistemically accessible world $w$ such that lying is permissible according to $\lesssim_{w}$; whether it is the actual world is immaterial.

\section{2. 'Must' and 'permissible'}

Kratzer informally glosses her semantics for modals as saying "that a proposition is a necessity if and only if it is true in all accessible worlds which come closest to the ideal established by the ordering source" $(1991,644)$. But her official semantics is a bit more complicated than this leads one to expect.

PM (Partial 'Must'):

'Must $\mathcal{C}$ ' is true at $i$ (relative to $\lesssim_{i}$ ) iff for every world $h \in S_{\varsigma_{i}}$ there is some world $j$ such that $j \lesssim_{i} h$ and every world $k$ such that $k \lesssim_{i} j$ is a $C$-world. (1981, 
$298 ; 1991,644)^{4}$

Two issues prompt the complications. First, Kratzer allows for preorders on which, for some $A$, for every $A$-world there is a better one. (In short, she does not endorse any analogues of the limit assumption.) Second, she wants to allow for preorders that are merely partial. The cases I use here to raise problems for Kratzer exploit these complications: they involve merely partial preorders on which an analogue of the limit assumption fails.

The status of the limit assumption and the importance of partiality are both controversial. But the controversy centers on the properties of the actual deontic ordering. For example, when Niko Kolodny and John MacFarlane defend the limit assumption for deontic modals, they write that it "is a safe assumption if (a) there can be only finitely many agents, (b) each agent can have only finitely many possible choices, and (c) no two worlds where agents make the same choices differ in respect of deontic ideality" $(2010,131)$. Whether or not these considerations establish anything about the properties of the actual deontic preorder, they are irrelevant to deontic modals embedded in constructions that shift the preorder. For example, I am not certain that there are finitely many agents facing only finitely many possible choices. Indeed, for all I know there are infinitely many agents facing infinitely many choices. So some preorders relevant to the evaluation of 'I must keep my promise' in (2) are infinite, even if the actual one is not.

(2) For all I know I must keep my promise.

Similarly for totality: as a matter of fact I suspect that the deontic preorder is partial. Whether or not I am right about this, however, I cannot imagine being certain that it is total. So for all I know the actual deontic preorder is merely partial. So even if the actual deontic preorder is total, some preorders relevant to evaluating 'I must keep my promise' in (2) will not be. Our semantics for 'must' and other modals should not be tailored to features of the actual deontic preorder. In light of our ignorance about the features of the actual deontic preorder, our semantics should not presuppose the limit assumption or any analogue of it, and should allow for merely partial preorders.

For brevity I will not go through the circumlocutions necessary to guarantee the semantic relevance of merely partial preorders that violate the limit assumption. Instead I will describe cases that, intuitively, call for such preorders in the characterization of a particular believer's deontic ordering, and I will tacitly assume that the believer is right about the deontic ordering. This last assumption is convenient

${ }^{4}$ I assume that the prejacents of modals and the antecedents and consequents of conditionals express propositions conceived of as sets of possible worlds, and I distinguish typographically between clauses $(\mathcal{A}, \mathcal{B}, \mathcal{C}, \ldots)$ and the propositions they express $(A, B, C, \ldots)$. 
but inessential: the reader is welcome to drop the assumption and instead embed sentences under, e.g., "Given the truth of $X$ 's beliefs about the deontic ordering" if it makes judgments easier to get. Moreover, the particular examples I will discuss are far less important than the principles they illustrate, so I invite the substitution of other illustrations of those principles wherever it is helpful.

Suppose that John and Karen deontically value proper supersets of children strictly increasingly, and that, because they think every life is uniquely precious, they think that sets of children neither of which is a subset of the other are deontically incomparable. They are able to choose to conceive exactly one of two potential children, Alice and Bert. This kind of situation calls for a preorder like that in

\section{Partiality 1:}

$S_{\varsigma_{i}}=\left\{i, w_{1}, w_{2}\right\} \cdot w_{1}<_{i} i, w_{2}<_{i} i, i \lesssim_{i} i, w_{1} \Varangle_{i} w_{2}, w_{2} \Varangle_{i} w_{1} \cdot w_{1}$ is a $C$-world, and $w_{2}$ and $i$ are $\neg C$-worlds.

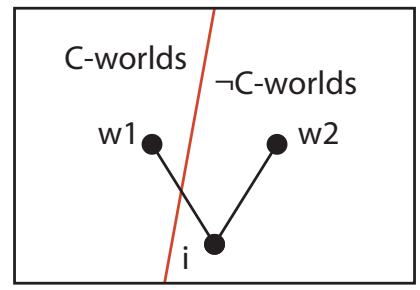

Partiality 1

Let $C$ be the proposition that Alice is conceived and Bert is not. Now consider the following semantics for 'must,' which is simpler than PM:

TM (Total 'Must'):

'Must $\mathcal{C}$ ' is true at $i$ (relative to $\lesssim_{i}$ ) iff some world $j$ in $S_{\aleph_{i}}$ is such that for every world $k$ in $S_{\aleph_{i}}$, if $k \lesssim_{i} j$ then $k$ is a $C$-world.

According to TM, 'Must $\mathcal{C}$ ' is true at $i$ because $w_{1} \in S_{\aleph_{i}}$ and for all worlds $k$ in $S_{\aleph_{i}}$ if $k \lesssim_{i} w_{1}$ then $k$ is a $C$-world. And 'Must not $\mathcal{C}$ ' is true at $i$ because $w_{2} \in S_{\varsigma_{i}}$ and for all worlds $k$ in $S_{\aleph_{i}}$ if $k \lesssim_{i} w_{2}$ then $k$ is a $\neg C$-world. Given that we model John and Karen's situation with a preorder like the one described in PARTIALITY 1, according to TM all of (3)-(6) are true at $i$.

(3) It must be that Alice is not conceived.

(4) It must be that Alice is conceived. 
(5) It must be that Bert is not conceived.

(6) It must be that Bert is conceived.

This is an unacceptable result. Intuitively, a conception is morally obligatory, contra (3) and (5). And it needn't be that Alice in particular is conceived, or that Bert in particular is conceived (contra (4) and (6)), although every way of improving on $i$ involves conceiving Alice or Bert. So TM is not an adequate semantics for 'must' in these cases. More generally, TM is not an adequate semantics for 'must' in cases that must be modeled with a merely partial preorder. I say this not to disparage $\mathrm{TM}-$ it was never intended to handle merely partial preorders-but only to show why the more complicated PM was proposed in the first place.

In contrast to TM, PM predicts that 'Must $\mathcal{C}$ ' will be false at $i$ given a model like Partiality 1. This is because for $h=w_{2}$, there is no world $j$ such that (i) $j \lesssim_{i} h$ and (ii) every world $k$ such that $k \lesssim_{i} j$ is a $C$-world. Similarly for 'Must not $\mathcal{C}$ ' and $h=w_{1}$. None of (3)-(6) come out true at $i$ according to PM. These are the intuitively correct predictions. Moreover, given that 'it must be that' and 'it is permissible that' are duals, all of (7)-(11) are true at $i$ according to PM.

(7) It is permissible that Alice is conceived.

(8) It is permissible that Alice is not conceived.

(9) It is permissible that Bert is conceived.

(10) It is permissible that Bert is not conceived.

(11) It must be that Alice or Bert is conceived.

Again these predictions are right. Every way of improving on $i$ involves conceiving Alice or Bert, and so it must be that Alice or Bert is conceived. Because Kratzer developed PM to make the right predictions for modals evaluated relative to partial preorders, I call it an example of partial preorder semantics for modals.

Note that Kratzer's semantics for 'Must $\mathcal{C}$ ' requires only that for each world $h \epsilon$ $S_{\aleph_{i}}$ there be some world $j$ such that $j \lesssim_{i} h$ and every world $k$ at least as good as $j$ is a $C$ world. It's not easy for there to be, for each such world $h$, some world $j$ satisfying both these conditions. So Kratzer's semantics is fairly strong. But it is not strong enough. For 'Must $\mathcal{C}$ ' may be true at $i$ according to PM even though $i$ can be improved on without limit without making $C$ true.

Here is an example. Add to what we already know about John and Karen that they have an unusual condition: they will have only boys unless they have an operation that will allow them to conceive one girl but will also make them infertile. They still deontically value finite proper supersets of children strictly increasingly but they 
believe they must have at most finitely many children. This deontic structure is represented in the figure below, labeled 'CHEAPER BY THE DOzEn.' The numbers indicate which children are conceived: boys have even numbers and girls have odd numbers.

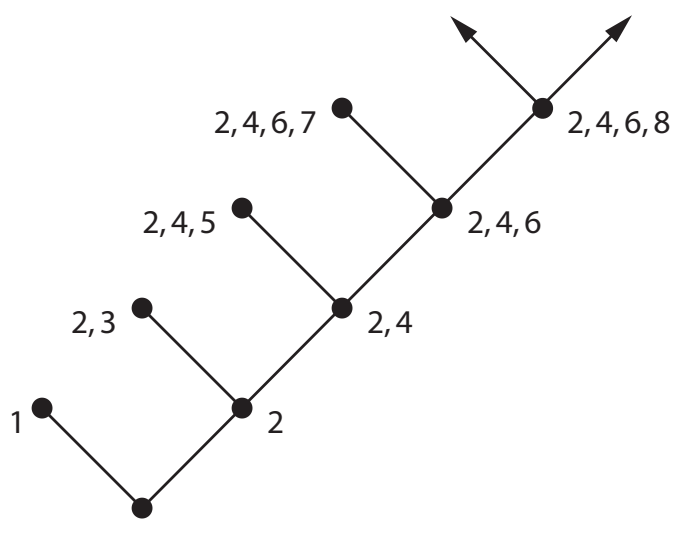

\section{CHEAPER BY THE DOZEN}

(12) and (13) are true at $i$ and (14) and (15) are false at $i$ according to PM. ${ }^{5}$

(12) It must be that a girl is conceived.

(13) It must be that the last child conceived is a girl.

(14) It is permissible that only boys are conceived.

(15) It must be that only boys are conceived.

The truth of (12) and (13) is very counterintuitive. According to the preorder, given that $n$ children will be conceived it is not better for child $2 n-1$ to be conceived, or to be conceived last, than it is for child $2 n$ to be conceived. The falsity of (14) is very counterintuitive for analogous reasons. And the difference between (12) and (15) is also counterintuitive: given the features of the preorder, girls do not have a special status that boys lack. Note also that the truth of (13) actually does not depend on the gender of even numbered children: it stays true even if if we omit that detail. Finally, note that (given Kratzer's views on conditionals) for any natural number $n$, (16) is true:

${ }^{5}$ It is worth noting that supervaluating over the partial preorder in the way prescribed by STALNAKER 1980, 89-91 and 1984, 134-135 and 140-142 (and endorsed by LeWIS 1981 and WEATHERSON 2001, among others) yields the correct results about this case: (12), (13), and (15) all are false at $i$, and (14) is true at $i$. Stalnaker's approach gives the wrong results about other cases, however; see my 2010. 
(16) If John and Karen were obligated to conceive at most $n$ children, they wouldn't have to conceive a girl.

The antecedent of (16) 'lops off' the top of the tree depicted in CHEAPER BY THE DOZEN, so that for some world $h \in S_{\varsigma_{i}}$ (namely, the best remaining solely evennumbered world) there is some world $j$ such that $j \lesssim_{i} h$ (namely, $h$ itself) and every world $k$ at least as good as $j$ is a world in which only boys are conceived. But the simultaneous truth of (12) and all the instances of (16) is very counterintuitive.

We need to refine partial preorder semantics. Some terminology will be helpful.

Definition 6. A set $S$ is $a \lesssim$ chain iff $\lesssim$ totally preorders $S$.

Although a chain is standardly understood to be a set with a total order (i.e., a set with an antisymmetric total preorder), here I allow chains and other standard order theoretic objects to be sets with mere preorders.

Definition 7. $A \lesssim$ chain $S$ is a maximal $\lesssim$ chain iff no $\lesssim$ chain properly includes $S$.

A straightforward adaptation of the Hausdorff maximality theorem ${ }^{6}$ to preorders guarantees that every $\lesssim$ chain is a subset of some maximal $\lesssim$ chain:

Proposition 1. For any partially preordered set $S$ and any totally preordered subset of $S$, $S^{\prime}$, there is a maximal totally preordered set $S^{\prime \prime}$ such that $S^{\prime} \subseteq S^{\prime \prime}$.

In other words, any $\lesssim$ chain can be 'extended' into a maximal $\lesssim$ chain. For example, in CHEAPER BY THE DOZEN, the chain that has as elements the world in which exactly child 2 is conceived and the world in which exactly children 2 and 4 are conceived can be extended into infinitely many different maximal chains. One such maximal chain has as elements the world in which exactly child 2 is conceived, the world in which exactly children 2 and 4 are conceived, and the world in which exactly children 2, 4, and 5 are conceived. (Another maximal chain terminates in the conception of child 7 , another in the conception of child 9, and so on.) But another such maximal chain has as elements the worlds in which John and Karen conceive only boys. Kratzer's semantics in effect disregards this maximal chain; it simply isn't relevant. All that Kratzer's semantics cares about in CHEAPER BY THE DOZEN and in similar cases is the fact that for each world in the modal base, there is some world that is at least as good in which John and Karen conceive a girl, with no better worlds than that one in which they do not conceive a girl.

Lewis's familiar metaphor of nested spheres centered on the world of evaluation $(1973,6-7)$ provides a helpful way to think of modals and counterfactuals when they are evaluated with respect to total preorders. No similarly helpful metaphor

${ }^{6}$ HausdorfF 1914, 140-141. For an excellent presentation see Kelley 1955, 31-36. 
has been given for partial preorders. But it is possible to think of a partially preordered set $S$ as being partitioned into an 'upper subset' containing all the elements that are at least as highly ranked as some element in a given 'lower bound, and a 'lower subset' containing the remaining elements of $S$. Reformulating PM in these terms helps bring out how certain relationships between a partial preorder and its maximal chains-relationships exemplified by CHEAPER BY THE DOZEN-can make trouble for PM. Moreover, this reformulation makes it easier to see how to treat cases like CHEAPER BY THE DOZEN correctly.

One way to partition a partially preordered set is with maximal antichains. $S$ is a $\lesssim$ antichain iff every element of $S$ is $\lesssim$ comparable to something, but no two elements of $S$ are $\lesssim$ comparable to each other.

Definition 8. A set $S$ is $a \lesssim$ antichain iff $\forall x(x \in S \rightarrow(\exists y(x \lesssim y \vee y \lesssim x) \wedge$ $\neg \exists z(z \in S \wedge x \lesssim z \vee z \lesssim x)))$.

Definition 9. $A \lesssim$ antichain $S$ is a maximal $\lesssim$ antichain iff no $\lesssim$ antichain properly includes $S$.

For example, the set of worlds in which John and Karen conceive a girl, in CHEAPER BY THE DOZEN, is a maximal antichain of that preorder. By an adaptation of Kurepa's antichain principle $\mathrm{e}^{7}$ to preorders, every preorder $\lesssim$ contains some maximal $\lesssim$ antichain:

Proposition 2. For any partially preordered set $S$, there is some maximal set of pairwise incomparable elements $S^{\prime}$ such that $S^{\prime} \subseteq S$.

In other words, every partially preordered set $S$ is a superset of a set of elements $S^{\prime}$ such that all the elements of $S^{\prime}$ are pairwise incomparable, and every element of $S$ is comparable to some element of $S^{\prime}$. It is via such sets $S^{\prime}$-maximal antichains-that Kratzer's semantics partitions sets of worlds into 'upper' and 'lower' subsets. This is because PM is equivalent to

AM (AnTichain 'Must'):

'Must $\mathcal{C}$ ' is true at $i$ (relative to $\lesssim_{i}$ ) iff there is some maximal $\lesssim_{i}$ antichain, $B$, such that $\forall h \forall j\left(\left(h \in B \wedge j \lesssim_{i} h\right) \rightarrow j \in C\right)$.

In English: 'Must $\mathcal{C}$ ' is true at $i$ (relative to $\lesssim_{i}$ ) iff there is some lower bound (construed as a maximal $\lesssim_{i}$ antichain) on $\lesssim_{i}$ such that every world at least as $\lesssim_{i}$ good as that lower bound is a $C$-world.

\footnotetext{
${ }^{7}$ Kurepa 1953. For an illuminating discussion see HALPERN 1972.
} 
Theorem 1. 'Must $\mathcal{C}$ ' is true at $i$ (relative to $\lesssim_{i}$ ) according to $P M$ iff it is true at $i$ (relative to $\lesssim_{i}$ ) according to $A M$.

Proof.

$\Rightarrow$ Let $T$ be the set of worlds such that $t \in T$ iff $\forall k\left(k \lesssim_{i} t \rightarrow k \in C\right)$. Suppose for reductio (i) that 'Must $\mathcal{C}$ ' is true at $i$ (relative to $\lesssim_{i}$ ) according to PM, so that $\forall h\left(h \in S_{\lesssim_{i}} \rightarrow \exists j\left(j \lesssim_{i} h \wedge \forall k\left(k \lesssim_{i} j \rightarrow k \in C\right)\right)\right)$, and (ii) that $T$ does not contain any maximal $\lesssim_{i}$ antichain $B$ such that $\forall h \forall j\left(\left(h \in B \wedge j \lesssim_{i}\right.\right.$ $h) \rightarrow j \in C)$. Then by the definition of 'maximal antichain', there is some world, $w$, such that $w \in S_{\varsigma_{i}}$ and $w$ is not $\lesssim_{i}$ comparable to any world in $T$. But for every world $h$ in $S_{\varsigma_{i}}$, some world in $T$ is at least as $\lesssim_{i} \operatorname{good}$ as $h$, because $\exists j\left(j \lesssim_{i} h \wedge \forall k\left(k \lesssim_{i} j \rightarrow k \in C\right)\right)$, and any such $j$ is a member of $T$. Contradiction. So $T$ does contain some maximal $\lesssim_{i}$ antichain $B$ such that $\forall h \forall j\left(\left(h \in B \wedge j \lesssim_{i} h\right) \rightarrow j \in C\right)$.

$\Leftarrow$ Suppose for conditional proof that 'Must $\mathcal{C}$ ' is true at $i$ (relative to $\lesssim_{i}$ ) according to AM, so that there is some maximal $\lesssim$ antichain, $B$, such that $\forall h \forall j\left(\left(h \in B \wedge j \lesssim_{i} h\right) \rightarrow j \in C\right)$. By the definition of 'maximal antichain', every world in $S_{\varsigma_{i}}$ is $\lesssim_{i}$ comparable to some world in $B$, since if a world in $S_{\lesssim_{i}}$ were not $\lesssim_{i}$ comparable to some world in $B$ then $B$ would not be a maximal $\lesssim_{i}$ antichain. If a world in $S_{\aleph_{i}}$ is at least as $\lesssim_{i}$ good as a world in $B$, then all the worlds at least as $S_{\aleph_{i}}$ good as it are $C$-worlds, by the construction of $B$ and the transitivity of $\lesssim_{i}$. If a world in $S_{\aleph_{i}}$ is not at least as $\lesssim_{i}$ good as any world in $B$, then there is some world that is $\lesssim_{i}$ better than it in $B$; without loss of generality let that world be $b$. All the worlds at least as $S_{\varsigma_{i}}$ good as $b$ are $C$-worlds, by the construction of $B$. So whether or not a given world in $S_{\varsigma_{i}}$ is at least as $\lesssim_{i}$ good as a world in $B, \forall h\left(h \in S_{\lesssim_{i}} \rightarrow \exists j\left(j \lesssim_{i} h \wedge \forall k\left(k \lesssim_{i} j \rightarrow k \in C\right)\right)\right)$.

A semantics that respects our intuitions about examples like CHEAPER BY THE DOZEN must take into account every maximal chain of any given preorder. A natural way to do this is to move from thinking of the lower bounds on partial preorders as maximal antichains to thinking of lower bounds on partial preorders as cutsets, instead.

Definition 10. A set $S$ is $a \lesssim$ cutset iff $S$ contains an element of each maximal $\lesssim$ chain. ${ }^{8}$

\footnotetext{
${ }^{8}$ For early work on cutsets see BELL \& GINSBURG 1984 (where, generalized to apply to graphs as well as to preorders, they are called "transversals") and GINSBURG 1984; see also GrILLET 1969. Some partially ordered sets lack minimal cutsets (Higgs 1985, LonC \& Rival 1987, and MALtBy 1994) so they are not good candidates to be lower bounds.
} 
CM (Cutset 'Must'):

'Must $\mathcal{C}$ ' is true at $i$ (relative to $\lesssim_{i}$ ) iff there is some $\lesssim_{i}$ cutset, $B$, such that $\forall h \forall j\left(\left(h \in B \wedge j \lesssim_{i} h\right) \rightarrow j \in C\right)$.

In English (and deliberately paralleling my earlier gloss of AM): 'Must $\mathcal{C}$ ' is true at $i$ (relative to $\lesssim_{i}$ ) iff there is some lower bound (construed as a $\lesssim_{i}$ cutset) on $\lesssim_{i}$ such that every world at least as $\lesssim_{i}$ good as that lower bound is a $C$-world.

The preorder depicted in CHEAPER BY THE DOzEN has many cutsets that are also maximal antichains. Here are three:

1. \{the world in which exactly child 1 is conceived, the world in which exactly child 2 is conceived $\}$;

2. \{the world in which exactly child 1 is conceived, the world in which exactly children 2 and 3 are conceived, the world in which exactly children 2 and 4 are conceived\};

3. the world in which exactly child 1 is conceived, the world in which exactly children 2 and 3 are conceived, the world in which exactly children 2, 4, and 5 are conceived, the world in which exactly children 2,4 , and 6 are conceived $\}$.

These sets are all cutsets because they intersect every maximal chain of the preorder. They are all maximal antichains because none of their elements are comparable to each other, by the lights of the relevant preorder, and every other world ordered by that preorder is comparable to at least one of them. However, the maximal antichain consisting of worlds in which a girl is conceived - \{the world in which exactly child 1 is conceived, the world in which exactly children 2 and 3 are conceived, the world in which exactly children 2,4 , and 5 are conceived, the world in which exactly children $2,4,6$, and 7 are conceived, ... - -is not a cutset, because it does not intersect every maximal chain of the preorder. In particular, it does not intersect the maximal chain \{the world in which exactly child 2 is conceived, the world in which exactly children 2 and 4 are conceived, the world in which exactly children 2, 4, and 6 are conceived, the world in which exactly children $2,4,6$, and 8 are conceived, ... $\}$. This is the heart of the difference between the AM/PM semantics for 'must' and the CM semantics. When some maximal antichain is 'better' than every cutset of a given preorder-in the sense that no world of the cutset is better than any world of the antichain, and some world of the antichain is better than some world of the cutset-the AM/PM semantics diverts its attention to that maximal antichain, treating it as a 'better' lower bound than any cutset lower bound.

Applying CM to CHeaper By THE DOzen provides a helpful illustration. (12) is false according to CM because there is no cutset such that every world at least as good as it is a world in which a girl is conceived. 
(12) It must be that a girl is conceived.

(13) is false according to CM because there is no cutset such that every world at least as good as it is a world in which a girl is the last child conceived.

(13) It must be that the last child conceived is a girl.

(14) is true according to CM (given, as before, that 'it must be that' and 'it is permissible that' are duals) because there is no cutset such that every world at least as good as it is a world in which a girl is conceived.

(14) It is permissible that only boys are conceived.

$\mathrm{CM}$ also rightly predicts that 'Must $\mathcal{C}$ ' is false at $i$ in PARTIALity 1.

Indeed we lose nothing in the move from $\mathrm{AM} / \mathrm{PM}$ to $\mathrm{CM}$, for they agree in all cases but some with crucial features of CHEAPER BY THE DOZEN. (I detail those cases in a moment.) To begin with, 'Must $\mathcal{C}$ ' is true according to $\mathrm{CM}$ only if it is true according to AM/PM.

Lemma 1. For each $\lesssim_{i}$ cutset $B$ there is some maximal $\lesssim_{i}$ antichain $A$ such that $A \subseteq B$.

Proof. Suppose for reductio that for some $\lesssim_{i}$ cutset $B$ there is no maximal $\lesssim_{i}$ antichain $A$ such that $A \subseteq B$. By Proposition 2 there is some maximal $\lesssim_{i}$ antichain. Because (by the reductio assumption) no maximal $\lesssim_{i}$ antichain is a subset of $B$, there is some element of $S_{\varsigma_{i}} \backslash B$ that is not $\lesssim_{i}$ comparable to any element of $B$; otherwise some subset of $B$ would be a maximal $\lesssim_{i}$ antichain. By Proposition 1 every element of $S_{\lesssim_{i}}$ is an element of some maximal $\lesssim_{i}$ chain, and so is comparable to every other element of that chain. So every element of $S_{\aleph_{i}}$ is $\lesssim_{i}$ comparable to some element of $B$, because $B$ contains an element of each maximal $\lesssim_{i}$ chain. Contradiction. So for each $\lesssim_{i}$ cutset $B$ there is some maximal $\lesssim_{i}$ antichain $A$ such that $A \subseteq B$.

Theorem 2. 'Must $\mathcal{C}$ ' is true at $i$ (relative to $\lesssim_{i}$ ) according to CM only if it is true at $i$ (relative to $\lesssim_{i}$ ) according to $A M$.

Proof. Suppose for conditional proof that 'Must $\mathcal{C}$ ' is true at $i$ (relative to $\lesssim_{i}$ ) according to CM, so that there is some $\lesssim_{i}$ cutset, $B$, such that $\forall h \forall j\left(\left(h \in B \wedge j \lesssim_{i} h\right) \rightarrow j \in C\right)$. By Lemma 1 there is some $\lesssim_{i}$ antichain, $A$, such that $A \subseteq B$. Because $A \subseteq B, \forall h \forall j((h \in$ $\left.A \wedge j \lesssim_{i} h\right) \rightarrow j \in C$ ). So 'Must $\mathcal{C}$ ' is true at $i$ (relative to $\lesssim_{i}$ ) according to AM.

The cases in which 'Must $\mathcal{C}$ ' is true according to AM/PM and not true according to $\mathrm{CM}$ have exotic features that Theorem 3, below, brings out. 
Lemma 2. As before, let $t \in T$ iff $\forall k\left(k \lesssim_{i} t \rightarrow k \in C\right)$. If 'Must $\mathcal{C}$ ' is not true at $i$ (relative to $\left.\lesssim_{i}\right)$ according to $C M$, then some maximal $\lesssim_{i}$ chain $M$ is such that $M \cap T=\varnothing$.

Proof. Suppose for reductio that 'Must $\mathcal{C}$ ' is not true at $i$ (relative to $\lesssim_{i}$ ) according to $\mathrm{CM}$ (so that there is no $\lesssim_{i}$ cutset, $B$, such that $\forall h \forall j\left(\left(h \in B \wedge j \lesssim_{i} h\right) \rightarrow j \in C\right)$ ) and that every maximal $\lesssim_{i}$ chain shares an element with $T$. By the assumption that 'Must $\mathcal{C}^{\prime}$ ' is not true at $i$ (relative to $\lesssim_{i}$ ) according to CM there is no $\lesssim_{i}$ cutset, $B$, such that $B \subseteq T$. But by the assumption that every maximal $\lesssim_{i}$ chain shares an element with $T$, there is some $\lesssim_{i}$ cutset that is a subset of $T$. Contradiction. So if 'Must $\mathcal{C}$ ' is not true at $i$ (relative to $\lesssim_{i}$ ) according to $\mathrm{CM}$, then some maximal $\lesssim_{i}$ chain $M$ is such that $M \cap T=\varnothing$.

Theorem 3. As before, let $t \in T$ iff $\forall k\left(k \lesssim_{i} t \rightarrow k \in C\right)$. If 'Must $\mathcal{C}$ ' is true at $i$ (relative to $\lesssim_{i}$ ) according to $A M$ and not according to $C M$, then there is some maximal $\lesssim_{i}$ chain $M$ and some maximal $\lesssim_{i}$ antichain $A$ such that $A \subseteq T$ and every element of $M$ is $\lesssim_{i}$ bettered by some element of $A$.

Proof. Suppose that 'Must $\mathcal{C}$ ' is true at $i$ (relative to $\lesssim_{i}$ ) according to AM and not according to CM. By the supposition that 'Must $\mathcal{C}$ ' is true at $i$ (relative to $\lesssim_{i}$ ) according to AM, there is some maximal $\lesssim_{i}$ antichain $A$ such that $A \subseteq T$. By the supposition that 'Must $\mathcal{C}$ ' is not true at $i$ (relative to $\lesssim_{i}$ ) according to CM and Lemma 2 there is some maximal $\lesssim_{i}$ chain $M$ such that $M \cap T=\varnothing$. Every element of $M$ is $\lesssim_{i}$ comparable to some element of $A$, because $M \subseteq S_{\aleph_{i}}$ and $A$ is a maximal $\lesssim_{i}$ antichain. Suppose that some element of $M$ is at least as $\lesssim_{i}$ good as some element of $A$. Then that element of $M$ is an element of $T$, by the supposition that $\forall h \forall j\left(\left(h \in A \wedge j \lesssim_{i} h\right) \rightarrow j \in C\right)$ and the transitivity of $\lesssim_{i}$. Contradiction. So every element of $M$ is $\lesssim_{i}$ bettered by some element of $A$.

So in any preorder that pulls the verdicts of AM/PM apart from those of CM, some maximal antichain beats every element of some maximal chain-as in CHEAPER BY THE DOZEN. Obviously this requires that the beaten maximal chain improve without end; if it contains a world that is not bettered by any worlds in the maximal chain itself then it will be impossible for a maximal antichain to beat every one of its elements. The presence of such a maximal chain is not a sufficient condition for 'Must $\mathcal{C}$ ' to be true according to AM/PM and untrue according to CM, of course: we also need that the beaten maximal chain does not 'settle' on $C$-worlds. But if any $C$-world in the beaten maximal chain is bettered by a $\neg C$-world in the maximal chain, 'Must $\mathcal{C}$ ' will not be true according to CM. This is consistent with every world in some maximal antichain being such that any world at least as good as it is a $C$-world, making 'Must $\mathcal{C}$ ' true according to AM/PM. This is precisely what we see in CHEAPER BY THE DOZEN. 
To capture our intuitions about that kind of case we need to use cutsets, not maximal antichains, as lower bounds in ordering semantics.

\section{3. 'Ought'}

The semantics for 'ought' on offer in VAN FrAASSEN 1973 is couched in terms that are very different from those of ordering semantics:

Suppose that $\beta$ is one of the possible alternatives we are considering. Let us say that the score of $\beta$ is the class of imperatives in force that $\beta$ fulfills. Then: 'It ought to be that $A$ ' is true if and only if there is a possible [accessible] state of affairs $\beta$ in $A$ whose score is not included in the score of any $\gamma$ in $\neg A$. (18, slightly paraphrased)

This can be seen as an early version of what came to be called premise semantics for modals, since it explicitly treats a modal as an expression that is sensitive to the relationship between the satisfaction of imperatives-which here play the role of 'premises' - and the content of its prejacent.'

One respect in which this semantics differs from Kratzer's ordering and premise semantics for 'must' is that van Fraassen's 'ought' does not validate agglomeration, and so allows for the consistency of moral dilemmas expressed with 'ought.' That is, on both $\mathrm{AM} / \mathrm{PM}$ and $\mathrm{CM}$, if it must be that $\mathcal{A}$ and it must be that $\mathcal{B}$, then it must be that $\mathcal{A}$ and $\mathcal{B}$. But on van Fraassen's semantics for 'ought', the fact that it ought to be that $\mathcal{A}$ and it ought to be that $\mathcal{B}$ does not entail that it ought to be that $\mathcal{A}$ and $\mathcal{B}$. So van Fraassen is in accord with E. J. Lemmon, who writes that

... an explicit contradiction is derivable from the assumption that a man both must and must not do something. But no similar contradiction is derivable from the assumption that someone both ought and ought not to do something .... It seems to me that "ought" and "ought not" may well both be true, and that this description in fact characterizes a certain class of moral dilemma. ...It is a nasty fact about human life the we sometimes both ought and ought not to do things; but it is not a logical contradiction. (1962, 149-150)

\footnotetext{
${ }^{9}$ The canonical works of early premise semantics (VeLtman 1976 and Kratzer 1976 and 1977) do not appear to have been directly influenced by this paper of van Fraassen's. For a prescient discussion of earlier work in a broadly similar vein, including CHISHOLm 1946 and GoodmaN 1947, see Lewis 1973, 65-72. Kratzer details her reasons for departing from the Chisholm/Goodman approach, and from Rescher's 1964 approach, in her 1979, 124-126.
} 
The invalidity of agglomeration is a desirable feature of van Fraassen's semantics for 'ought.'

Unfortunately his semantics makes counterintuitive predictions about CHEAPER BY THE DOZEN, just as Kratzer's ordering semantics does. Suppose that the set of imperatives in force is

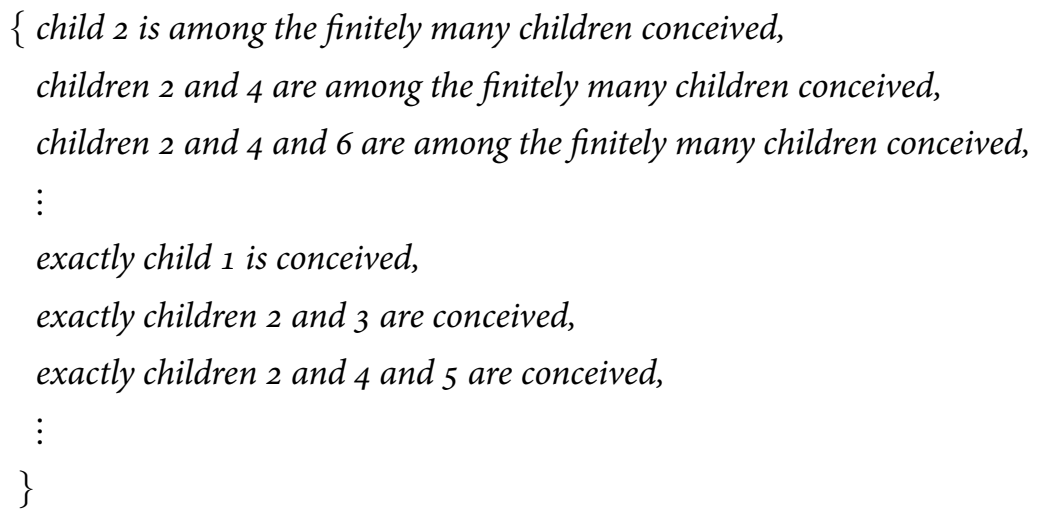

Then the score of any possible alternative $\beta$ is a subset of the score of some possible alternative in which an odd numbered child - a girl—is conceived. So there is no $\beta$ in which only boys are conceived the score of which is not included in any $\gamma$ in which a girl is conceived. So (17) comes out true on this semantics, but (18) comes out false.

(17) It ought to be that the last child conceived is a girl.

(18) It ought to be that the last child conceived is a boy.

The intuitive prediction, I think, is that both these sentences are true. In short, by John and Karen's lights, it is not the case for any $n$ that the conception of girl $2 n-1$ has more deontic worth than the conception of boy $2 n$. But this is not consistent with rendering (17) true and (18) false. John and Karen have found themselves in a moral dilemma-really, in infinitely many moral dilemmas. It is impossible for all the infinitely many mutually inconsistent things that ought to be true to be true.

Given the translation between ordering frames and premise sets described in Lewis $1981,{ }^{10}$ Kratzer's ordering semantics for 'is a good possibility' $(1991,644)$ is equivalent to van Fraassen's semantics for 'ought' (and, incidentally, to TM, construed as a semantics not for 'must' but for 'ought'). All these semantics are equivalent to

\footnotetext{
${ }^{10}$ Jean-Paul Doignon and Jean-Claude Falmagne provide a rich set of related results in their 1985 and 1999. Their work was (and, unfortunately, remains) independent from work in ordering semantics and premise semantics per se.
} 
OSO (Ordering Semantics 'Ought'):

'Ought $\mathcal{C}$ ' is true at $i$ (relative to $\lesssim_{i}$ ) iff there is some world $j$ such that $j \lesssim_{i} i$ and every world $k$ such that $k \lesssim_{i} j$ is a $C$-world.

I suggest that we replace this semantics for 'ought' with

MCO (Maximal Chain 'Ought'):

'Ought $\mathcal{C}$ ' is true at $i$ (relative to $\lesssim_{i}$ ) iff 'Must $\mathcal{C}$ ' is true at $i$ relative to some maximal $\lesssim_{i}$ chain.

(17) still comes out true on MCO: every maximal $\lesssim_{i}$ chain that ends in a world in which the last child conceived is a girl is, on any plausible ordering semantics for 'must,' a maximal $\lesssim_{i}$ chain relative to which 'It must be that the last child conceived is a girl' is true. But now (18) comes out true as well, because 'It must be that the last child conceived is a boy' is true relative to the maximal $\lesssim_{i}$ chain consisting of worlds in which only boys are conceived.

The fundamental thought here is that weak necessity modals like 'ought' and 'should' abstract away from incomparability: 'Ought $\mathcal{C}$ ' is true iff there is some way of bracketing moral dilemmas on which 'Must $\mathcal{C}$ ' is true. More formally put, and within the framework of ordering semantics, weak necessity modals decompose partial preorders into their constituent maximal chains and test those maximal chains against the standards associated with strong necessity modals like 'must' and 'have to.' This is certainly not the only difference between weak and strong necessity modals. But it is plausible that it is at least part of the explanation of why (17) and (18) are consistent even though (13) and (19) are inconsistent.

(13) It must be that the last child conceived is a girl.

(19) It must be that the last child conceived is a boy.

Recent accounts of the distinction between weak and strong necessity modals-in particular, those advocated by Kratzer 1991, ${ }^{11}$ Sæв $\varnothing ~ 2001$, von Fintel \& IATrIDOU 2005, Copley 2006, von Stechow et al. 2006, and von Fintel \& IATridou 2008-wrongly render (17) and (18) every bit as inconsistent as (13) and (19).

This idea can also be easily expressed within the framework of premise semantics. A set of van Fraassen's scores-where, again, a score is just a set of imperatives-may be $\mathrm{a} \subseteq$ chain. $\mathrm{A} \subseteq$ chain of scores represents a direction of improvement; a maximal $\subseteq$ chain of scores represents a maximally specified direction of improvement. The analogue of MCO in van Fraassen's terminology simply says that 'Ought $\mathcal{C}$ ' is true if

\footnotetext{
${ }^{11}$ Kratzer's semantics there is equivalent to the semantics van Fraassen considers and rejects in his 1973,7 .
} 
and only if 'Must $\mathcal{C}$ ' is true relative to a maximal $\subseteq$ chain of scores. More intuitively, 'Ought $\mathcal{C}$ ' is true if and only if 'Must $\mathcal{C}$ ' is true relative to some maximally specified direction of improvement. On the premise semantic way of thinking that Kratzer and Veltman develop, a modal is evaluated relative to a set of premises-the premise set. Call each consistent subset of a premise set $P$ an argument associated with $P$. Expressed in these terms, MCO says that 'Ought $\mathcal{C}$ ' is true if and only if 'Must $\mathcal{C}$ ' is true relative to a maximal $\subseteq$ chain of arguments. And on the original Kratzer/Veltman semantics-equivalent to $\mathrm{PM}-$ 'Must $\mathcal{C}$ ' is true relative to a premise set $P$ iff every argument $A$ associated with $P$ can be 'extended' to some argument $B$ associated with $P$ such that $B$ establishes that $\mathcal{C}$. (An argument $B$ extends an argument $A$ iff $A \subseteq B$.) Intuitively, 'Must $\mathcal{C}$ ' is true iff every relevant argument can be extended to a relevant argument that establishes that $\mathcal{C}$. On the premise semantic analogue of $\mathrm{CM}$, by contrast, 'Must $\mathcal{C}$ ' is true relative to a premise set $P$ iff every maximal chain of relevant arguments contains an argument that establishes that $\mathcal{C}$. Intuitively, the premise semantic analogue of CM asks whether every direction along which arguments constructed out of the premise set might be elaborated is a direction that establishes that $\mathcal{C}$. 'Must $\mathcal{C}$ ' is true iff this condition is satisfied.

\section{Counterfactuals and deontic conditionals}

We are now in a good position to assess the treatment of incomparability in David Lewis's work on counterfactuals. The Lewis Shell, as I call it here, is a characteristic feature of Lewisian theories of counterfactuals and deontic conditionals. It in effect asks us to evaluate ' $\mathcal{A}>\mathcal{C}$ ' by ignoring $\neg A$-worlds (often including $i$ itself) and asking whether 'Must $\mathcal{C}$ ' is true at $i$. The antecedent restricts the preorder used to evaluate 'Must $\mathcal{C}$ ' to preorder only the $A$-worlds in $S_{\aleph_{i}}$ (cf. Lewis 1975, 9-11; KRATZER 1981, 318-319 and 1991, 648-649). The assumption that $A$ is a set of worlds allows for an elegant implementation.

Definition 11. $A^{2}$ (read 'the Cartesian square of $\left.A^{\prime}\right)=\{\langle x, y\rangle: x \in A \wedge y \in A\}$

A partial preorder $\lesssim$ is just a relation, modeled by a set of ordered pairs. So by intersecting a preorder $\lesssim$ with another set of ordered pairs we can generate an order preserving subpreorder. In particular, intersecting $\lesssim_{i}$ with $A^{2}$ yields a preorder over exactly the $A$-worlds preordered by $\lesssim_{i}$ that agrees with $\lesssim_{i}$ on the relations between them. To improve readability I abbreviate ' $\aleph_{i} \cap A^{2}$ ' with ' $\aleph_{i}^{A}$, , and (as before) ' $\left\{w: w \lesssim_{i}^{A} i \vee i \lesssim_{i}^{A} w\right\}$ ' with ' $S_{\aleph_{i}^{A}}$ '. Thus we have the

LEWIS SHELL:

' $\mathcal{A}>\mathcal{C}$ ' is true at $i$ relative to $\lesssim_{i}$ iff'Must $\mathcal{C}$ ' is true at $i$ relative to $\lesssim_{i}^{A}$, or $\lesssim_{i}^{A}=\varnothing$. 
Obviously the Lewis Shell yields a semantics for ' $\mathcal{A}>\mathcal{C}$ ' only once it is supplemented with a semantics for 'Must $\mathcal{C}$.' This makes it quite illuminating for present purposes. By factoring out the contribution the Lewis Shell makes to Lewis's 1973 and 1981 semantics, we can see the differences between those semantics as differences in the semantics of 'Must $\mathcal{C}$.' Here is Lewis's 1973 semantics, and a reformulation that exposes the role of the Lewis Shell.

TC (Total Counterfactuals/Deontic Conditionals):

' $\mathcal{A}>\mathcal{C}$ ' is true at $i$ (relative to $\lesssim_{i}$ ) iff if there is an $A$-world in $S_{\aleph_{i}}$, then there is some $A$-world $j$ in $S_{\aleph_{i}}$ such that for all $A$-worlds $k$, if $k \lesssim_{i} j$ then $k$ is a $C$-world $(1973,49)$.

$\mathrm{TC}^{\prime}:$

' $\mathcal{A}>\mathcal{C}$ ' is true at $i$ (relative to $\lesssim_{i}$ ) iff according to TM 'Must $\mathcal{C}$ ' is true at $i$ relative to $\lesssim_{i}^{A}$, or $\lesssim_{i}^{A}=\varnothing$.

Theorem 4. ' $\mathcal{A}>\mathcal{C}$ ' is true according to $T C$ iff it is true according to $T C^{\prime}$.

Proof.

$\Rightarrow$ Suppose ' $\mathcal{A}>\mathcal{C}$ ' is true at $i$ (relative to $\lesssim_{i}$ ) according to TC. The vacuous case is trivial. In the non-vacuous case there is some $A$-world $j$ in $S_{\aleph_{i}}$ such that for all $A$-worlds $k$, if $k \lesssim_{i} j$ then $k$ is a $C$-world. ' $\mathcal{A}>\mathcal{C}$ ' is true at $i$ (relative to $\lesssim_{i}$ ) according to $\mathrm{TC}^{\prime}$ because there is some world $j^{\prime}$ in $S_{\varsigma_{i}^{A}}$ (namely $j$ ) such that for all worlds $k^{\prime}$ in $S_{\varsigma_{i}^{A}}$, if $k^{\prime} \lesssim_{i}^{A} j^{\prime}$ then $k^{\prime}$ is a $C$-world, since $\lesssim_{i}^{A}$ is a subpreorder of $\lesssim_{i}$.

$\Leftarrow$ Suppose ' $\mathcal{A}>\mathcal{C}$ ' is true at $i$ (relative to $\lesssim_{i}$ ) according to $\mathrm{TC}^{\prime}$. The vacuous case is trivial. In the non-vacuous case, the truth of 'Must $\mathcal{C}$ ' according to TM entails that there is some world $j$ in $S_{\aleph_{i}^{A}}$ such that for all worlds $k$ in $S_{\aleph_{i}^{A}}$, if $k \lesssim_{i}^{A} j$ then $k$ is a $C$-world. ' $\mathcal{A}>\mathcal{C}$ ' is true at $i$ (relative to $\lesssim_{i}$ ) according to TC because there is some $A$-world $j^{\prime}$ in $S_{\varsigma_{i}}$ (namely $j$ ) such that for all $A$ worlds $k^{\prime}$, if $k^{\prime} \lesssim_{i} j^{\prime}$ then $k^{\prime}$ is a $C$-world, since $\lesssim_{i}^{A}$ preorders all the $A$-worlds preordered by $\lesssim_{i}$.

Given the role that TM plays in $\mathrm{TC}^{\prime}$ and Theorem 4, it should not be surprising that TC makes bad predictions when asked to operate on partial preorders of worlds similar to those that were a problem for TM. For example: 


\section{PARTiality 2:}

The set of worlds $\lesssim_{i}$-comparable to $i$ is $\left\{i, w_{1}, w_{2}\right\} . w_{1}<_{i} i, w_{2}<_{i} i, i \lesssim_{i} i$, $w_{1} \Varangle_{i} w_{2}, w_{2} \Varangle_{i} w_{1} \cdot w_{1}$ is an $(A \wedge C)$-world, and $w_{2}$ is an $(A \wedge \neg C)$-world.

According to TC, ' $\mathcal{A}>\mathcal{C}^{\prime}$ ' is true in $i$, thanks to $w_{1}$. This prediction is wrong for a familiar reason: at some non-bettered $A$-world comparable to $i-$ namely, $w_{2}-C$ is false. As Lewis puts it, TC "might misevaluate a counterfactual as false ... because the worlds where the antecedent holds divide into incomparable classes" (1981, 230).

Lewis's 1981 semantics avoids this result.

PC (Partial Counterfactuals/Deontic Conditionals): ' $\mathcal{A}>\mathcal{C}$ ' is true at $i$ (relative to $\lesssim_{i}$ ) iff for every $A$-world $h$ comparable to $i$, there is some $A$-world $j$ such that (i) $j \lesssim_{i} h$ and (ii) every $A$-world $k$ such that $k \lesssim_{i} j$ is also a $C$-world. (230)

' $\mathcal{A}>\mathcal{C}$ ' is false at $i$ according to PC because $w_{2}$ (an $A$-world comparable to $i$ ) is such that the only $A$-world $j$ such that $j \lesssim_{i} w_{2}$ is $w_{2}$ itself, but some $A$-world $k$ such that $k \lesssim_{i} j$ is not a $C$-world, since $w_{2} \lesssim_{i} w_{2}$, and $w_{2}$ is a $\neg C$-world.

PC can be expressed using the Lewis Shell as follows:

$\mathrm{PC}^{\prime}:$

' $\mathcal{A}>\mathcal{C}$ ' is true at $i$ (relative to $\lesssim_{i}$ ) iff according to PM 'Must $\mathcal{C}$ ' is true at $i$ relative to $\lesssim_{i}^{A}$, or $\lesssim_{i}^{A}=\varnothing$.

Theorem 5. ' $\mathcal{A}>\mathcal{C}$ ' is true according to $P C$ iff it is true according to $P C^{\prime}$.

Proof.

$\Rightarrow$ Suppose ' $\mathcal{A}>\mathcal{C}$ ' is true at $i$ (relative to $\lesssim_{i}$ ) according to PC. The vacuous case is trivial. In the non-vacuous case for every $A$-world $h$ comparable to $i$, there is some $A$-world $j$ such that (i) $j \lesssim_{i} h$ and (ii) every $A$-world $k$ such that $k \lesssim_{i} j$ is also a $C$-world. ' $\mathcal{A}>\mathcal{C}$ ' is true at $i$ (relative to $\lesssim_{i}$ ) according to $\mathrm{PC}^{\prime}$ because for every world $h \lesssim_{i}^{A}$-comparable to $i$ there is some world $j^{\prime}$ in $S_{\varsigma_{i}^{A}}$ such that $j^{\prime} \lesssim_{i}^{A} h$ and every world $k^{\prime}$ in $S_{\aleph_{i}^{A}}$ such that $k^{\prime} \lesssim_{i}^{A} j^{\prime}$ is a $C$-world, since $<_{i}^{A}$ is a subpreorder of $\lesssim_{i}$.

$\Leftarrow$ Suppose ' $\mathcal{A}>\mathcal{C}$ ' is true at $i$ (relative to $\lesssim$ ) according to $\mathrm{PC}^{\prime}$. The vacuous case is trivial. In the non-vacuous case, the truth of 'Must $\mathcal{C}$ ' according to TM entails that for every world $h$ comparable to $i$, there is some world $j$ such that (i) $j \lesssim_{i}^{A} h$ and (ii) every world $k$ such that $k \lesssim_{i}^{A} j$ is a $C$-world. ' $\mathcal{A}>\mathcal{C}$ ' is true at $i$ (relative to $\lesssim_{i}$ ) according to PC because for every $A$-world $h$ comparable 
to $i$, there is some $A$-world $j^{\prime}$ such that (i) $j^{\prime} \lesssim_{i} h$ and (ii) every $A$-world $k^{\prime}$ such that $k^{\prime} \lesssim_{i} j$ is also a $C$-world, since $\lesssim_{i}^{A}$ preorders all the $A$-worlds preordered by $\lesssim_{i}$.

Unsurprisingly, PC and PM founder in similar ways on similar examples. It is harder to design preorders for counterfactuals than it is for deontic modals, because the preorders for counterfactuals must conform to weak centering (at least) and must respect our intuitions, such as they are, about minimal change. It may be that there are no reasonably natural sounding non-deontic counterfactuals the evaluation of which requires a structure like CHEAPER BY THE DOzen. For deontic conditionals, which Lewis discusses at length in chapter 5 of Counterfactuals, things are considerably easier. PC makes all of the following true, given a background of deontic value strictly increasing with proper supersets of children:

(20) Given that finitely many boys and at most one girl are conceived, a girl must be conceived.

(21) Given that finitely many boys and at most one girl are conceived, with any girl conceived last, the last child conceived must be a girl.

(22) If it had to be that finitely many boys and at most one girl were conceived, then a girl would have to be conceived.

(23) If it had to be that finitely many boys and at most one girl were conceived, with any girl conceived last, the last child conceived would have to be a girl.

Replacing PM with CM in the Lewis Shell yields a semantics that is recognizably Lewisian, in a broad sense, and that makes the right predictions about (20)-(23).

CC (Cutset Counterfactuals/Deontic Conditionals):

' $\mathcal{A}>\mathcal{C}$ ' is true at $i$ (relative to $\lesssim_{i}$ ) iff there is some $\lesssim_{i}^{A}$ cutset, $B$, such that $\forall h \forall j\left(\left(h \in B \wedge j \lesssim_{i}^{A} h\right) \rightarrow j \in C\right)$.

$\mathrm{CC}^{\prime}:$

' $\mathcal{A}>\mathcal{C}$ ' is true at $i$ (relative to $\lesssim_{i}$ ) iff according to CM 'Must $\mathcal{C}$ ' is true at $i$ relative to $\lesssim_{i}^{A}$, or $<_{i}^{A}=\varnothing$.

Together, Theorems 2 and 3 and the Lewis Shell's exposure of the similarities between PC and CC make it easy to see that Corollary 1 is true:

Corollary 1. Let $t \in T$ iff $\forall k\left(k \lesssim_{i}^{A} t \rightarrow k \in C\right)$. PC and CC agree on the truth value of ' $\mathcal{A}>\mathcal{C}$ ' at $i$ (relative to $\lesssim_{i}$ ) unless there is some maximal $\lesssim_{i}^{A}$ chain $M$ and some maximal 
$\lesssim_{i}^{A}$ antichain $B$ such that $B \subseteq T$ and every element of $M$ is $\lesssim_{i}^{A}$ bettered by some element of $B$.

Just as CM strictly improves on PM, CC strictly improves on PC. A theorist who endorses PC (but restricts it to ordinary counterfactuals) and endorses CC (but restricts it to deontic conditionals) is in a pretty precarious position. Absent strong arguments to the contrary, endorsing CC for both ordinary counterfactuals and deontic conditionals seems the more sensible route.

\section{References}

Bell, Murray \& John Ginsburg. 1984. "Compact Spaces and Spaces of Maximal Complete Subgraphs." Transactions of the American Mathematical Society, vol. 283 (1): $329-338$.

Chisholm, Roderick M. 1946. “The Contrary-to-Fact Conditional.” Mind, vol. 55: $289-307$.

Copley, Bridget. 2006. "What Should Should Mean?” Ms., CNRS/Université Paris 8, URL http://copley.free.fr/copley.should.pdf.

Doignon, Jean-Paul \& Jean-Claude Falmagne. 1985. "Spaces for the Assessment of Knowledge." International Journal of Man-Machine Studies, vol. 23 (2): 175-196.

-. 1999. Knowledge Spaces. Springer, Berlin.

von Fintel, Kai \& Sabine Iatridou. 2005. "What to Do If You Want to Go to Harlem: Anankastic Conditionals and Related Matters." Ms., Massachusetts Institute of Technology.

-. 2008. "How to Say Ought in Foreign: The Composition of Weak Necessity Modals." In Time and Modality, JaCqueline Guéron \& JaCqueline LeCarme, editors, vol. 75 of Studies in Natural Language and Linguistic Theory, 115-141. Springer.

van Fraassen, Bas C. 1973. "Values and the Heart's Command." Journal of Philosophy, vol. 70 (1): 5-19.

Ginsburg, John. 1984. "Compactness and Subsets of Ordered Sets That Meet All Maximal Chains.” Order, vol. 1: 147-157.

Goodman, Nelson. 1947. "The Problem of Counterfactual Conditionals." Journal of Philosophy, vol. 44 (5): 113-128. 
Grillet, Pierre Antoine. 1969. "Maximal Chains and Antichains." Fundamenta Mathematicae, vol. 65: 157-167.

Hájek, Alan. 2009. "Most Counterfactuals Are False." Ms., Australian National University.

Halpern, J. D. 1972. "On a Question of Tarski and a Maximal Theorem of Kurepa." Pacific Journal of Mathematics, vol. 41 (1): 111-121.

Hausdorff, Felix. 1914. Grundzüge der Mengenlehre. von Veit, Leipzig.

Higgs, Denis. 1985. "A Companion to Grillet's Theorem on Maximal Chains and Antichains." Order, vol. 1: 371-375.

Kelley, John L. 1955. General Topology. van Nostrand, New York.

Kolodny, Niko \& John MacFarlane. 2010. "Ifs and Oughts." Journal of Philosophy, vol. 107 (3): 115-143.

Kratzer, Angelika. 1976. “Was 'Können’ und 'Müssen’ Bedeuten Können und Müssen.” Linguistische Berichte, vol. 42.

-. 1977. "What Must and Can Must and Can Mean." Linguistics and Philosophy, vol. 1: 337-355.

-. 1979. "Conditional Necessity and Possibility." In Semantics from Different Points of View, R. Bauerle, U. Egli \& A. von Stechow, editors, 117-147. Springer-Verlag.

-. 1981. "The Notional Category of Modality." In Formal Semantics: The Essential Readings, Paul Portner \& Barbara H. Partee, editors, 289-323. Blackwell Publishers Ltd., Oxford.

-. 1991. "Modality." In Semantics: An International Handbook of Contemporary Research, Arnim von Stechow \& Dieter Wunderlich, editors, 639-650. W. de Gruyter, Berlin.

Kurepa, Đuro. 1953. "Über das Auswahlaxiom.” Mathematische Annalen, vol. 126: $381-384$.

Lemmon, E. J. 1962. “Moral Dilemmas.” Philosophical Review, vol. 71 (2): 139-158.

Lewis, David K. 1973. Counterfactuals. Basil Blackwell Ltd., Malden, MA.

-. 1975. "Adverbs of Quantification." In Formal Semantics of Natural Language, Edward L. Keenan, editor, 3-15. Cambridge University Press, Cambridge. 
-. 1981. "Ordering Semantics and Premise Semantics for Counterfactuals." Journal of Philosophical Logic, vol. 10: 217-234.

Lonc, Zbigniew \& Ivan Rival. 1987. "Chains, Antichains, and Fibres." Journal of Combinatorial Theory, Series A, vol. 44: 207-228.

Maltby, Roy. 1994. "When Is Every Minimal Cutset an Antichain?" Acta Scientiarum Mathematicarum, vol. 59: 381-403.

Rescher, Nicholas. 1964. Hypothetical Reasoning. North Holland, Amsterdam.

SÆвø, KJell Johan. 2001. "Necessary Conditions in a Natural Language.” In $A u$ diatur Vox Sapientiae: A Festschrift for Arnim von Stechow, CAROLIne FÉRY \& WolfGANG STERNEFELD, editors, vol. 52 of Studia Grammatica, 427-449. Akademie Verlag, Berlin.

Stalnaker, Robert C. 1980. "A Defense of Conditional Excluded Middle." In Ifs: Conditionals, Belief, Decision, Chance, and Time, William L. Harper, Robert Stalnaker \& Glenn Pearce, editors, 87-104. D. Reidel Publishing Company, Dordrecht.

-. 1984. Inquiry. MIT Press, Cambridge.

von Stechow, Arnim, Sveta Krasikova \& Doris Penka. 2006. "Anankastic Conditionals Again.” In A Festschrift for Kjell Johan Sxebø-In Partial Fulfillment of the Requirements of the Celebration of his 5oth Birthday, Atre Grønn, Dag Haug \& TORGRIM SolSTAD, editors, 151-171. Oslo University, Oslo.

Swanson, ERIC. 2010. "Ordering Supervaluationism, Counterpart Theory, and Ersatz Fundamentality." Ms., University of Michigan Ann Arbor.

Veltman, Frank. 1976. "Prejudices, Presuppositions and the Theory of Conditionals." In Amsterdam Papers in Formal Grammar, Volume 1, Jeroen Groenendijk \& MARTIN StокноF, editors, 248-281. Central Interfaculteit, University of Amsterdam, Amsterdam.

Weatherson, Brian. 2001. "Indicative and Subjunctive Conditionals." The Philosophical Quarterly, vol. 51 (203): 200-216. 